\title{
Robot Telescopes: a new era in access to astronomy
}

\author{
By J.E.F. Baruch \\ Department of Industrial Technology, University of Bradford, Bradford BD7-1DP
}

\section{Background}

For the teaching of astronomy there can be no alternative to the hands-on experience of using instruments on a real telescope observing on a clear dark night. Such experience is not possible for millions of students who are excited by the ideas of astronomy. It is not merely one of cost. The logistics of assembling a class of students after school hoping for clear skies destroys the possibilities of real observing for the majority of students. Robot telescopes change all that.

In educational terms a robot telescope can provide a range of experiences of observational astronomy. The development of CD-ROM and the Internet to support classroom learning have produced the concept of REAL(Dunlap 1996): a Rich Environment for Active Learning as an appropriate framework on which to develop the classroom response to these technologies. The Bradford Robot Telescope has demonstrated student centred experiences to generate a Rich Environment for Active Learning(REAL), for astronomy. It is based on a massive extension of the library and experiential resource available to the teacher over the Internet, the opportunity for the student to develop and answer questions associated with the learning programme and access to a robot telescope which provides two modes of operation: service observing and eavesdropping. In the concept of REAL the students are:-

- Allowed to, and taught to, determine what they need to learn through questioning and goal setting

- Provided with sufficient scaffolding in the environment to help students with prompts, examples, modelling and collaborative support

- Enabled to manage their own learning activities

- Enabled to contribute to each others' learning through collaborative activities.

The objective of the schools programme of the Bradford Robot Telescope is to support REAL environments in the classroom.

\section{The Bradford Robot Telescope}

The development of robot systems for astronomy has a long history. In 1955 Bart J. Bok (Bok 1955) called for the development of a "small automatic monitoring telescope which would provide at all times exact extinction information and provide valuable yearly information concerning the photoelectric properties of the sky". Vincent Reddish (Reddish 1966) built an automatic system to provide standards for photographic plates and in the mid sixties the US National Academy of Sciences produced a ten-year programme for ground based astronomy with a central proposal that significant funds be allocated to the development of automated telescopes and instrumentation such as plate measuring machines (Whitford 1964). In the end these programmes were suffocated through technological problems (Baruch 1992) which have now been solved with the Bradford system.

Towards the end of the Eighties the UK Science and Engineering Research Council (SERC) produced a Ground Based Plan for Astronomy 1990 (Mitchell 1989). This proposed the construction of small robot telescopes. By the early ' 90 's there were a growing number of problems that robot telescopes could make a significant contribution to solving:

- Scientific investments in satellite observatories operating in wavebands outside the visible were being compromised by the inability to follow up observations and more importantly to perform concurrent observations in the visible wavebands. Important science was being lost 
- The limited ability to respond to targets of opportunity e.g. comets, novae, supernovae and cataclysmic variables, was a loss to science

- The award of observing time on telescopes was largely based on the potential returns measured by the number of publications arising from the observations. The science which requires the frequent and long term monitoring of variable objects was not being pursued

- Surveys of particular types were limited and patrols searching for particular events were virtually non-existent

- There were few if any searches for planetary systems outside the solar system

- Within the solar system there was much uncertainty about the science of the satellites of the outer planets and of the asteroids and comets.

Arising from the Ground Based Plan the SERC initiated the development of a robot telescope by Baruch and his team.

The telescope was constructed at the Bradford University Experimental site near Haworth in the Pennines about 15 kilometres from the University Campus. It consists of a local network of telescope control computers at the observatory, linked to the main campus via an ISDN telephone line. The control computers are called Control, Point, Image, Weather and Catalogue each with the appropriate functions. The telescope is an alt-az Newtonian with the two major foci taken through the altitude bearings. The telescope is primarily for CCD photometry and supports a CCD photometer with broad band BVRI\&Z filters. A major objective of the telescope design was to reduce telescope costs to a small fraction of the current cost of a 1 metre telescope. The telescope is designed as a robot which can be assembled by two people on the top of a mountain without heavy lifting gear and this limits the aperture to about $1.2 \mathrm{~m}$ with a hextek type mirror.

The Bradford Robot has a $46 \mathrm{~cm}$ mirror. The CCD has a field of about 12 by 20 arc minutes. The telescope design allows for two main instrument locations for large heavy instruments and two subsidiary instrument locations. A mirror is rotated to move the focus between the different locations. The major software components are identical with systems used on large telescopes or are commercial software packages modified where necessary to meet the less demanding requirements of $1 \mathrm{~m}$ aperture robot telescopes.

The telescope is accessed over the Internet via the base station (Baldrick) which is located at the main University campus. The address of Baldrick and the telescope is http://www.telescope.org/ Access to Baldrick on the World Wide Web is via any one of the Mosaic or Netscape type of browsers currently available for a number of platforms including Suns and PCs. The user is then presented with a list of hypertext choices. They can read an on line guide to the telescope, find out technical details of the hardware and software, learn more about stars and galaxies, read weather reports, and control the telescope, all from the same interface.

The detailed operation of the telescope is described by Baruch (1996).

Baldrick presents visitors with a set of links which include: Access to the telescope, the Nuffield Research Project, Selected Internet References for Schools, Stars and Galaxies a CDROM conversion with graphics, pictures and hypertext links to provide a basic information resource in astronomy. Apart from the astronomy resource material the telescope has demonstrated two significant active classroom resources: service observing and eavesdropping.

The normal access to the telescope provides service observing. The user can requests an observation by selecting it from a browsable list with hyperlinks to automatically set up the request. The lists include solar system objects, a list of stars by name, the Messier Catalogue and IC, SAO and NGC catalogues. The user can also make a request for an observation of a specific RA and dec. The interface is designed to be helpful and supportive with comments and default values always available. For the least sophisticated user clicking on the mouse when pointing at the name of the desired object will set the system up to take an image of this object and inform the user by email when the image has been taken.

Users are requested to register by entering their username and password. In this way the system can allocate them user space on the system, return a notice that their observations has been made and the users can look at the progress of their observing request on the data base. An important aspect of the observing process is the generation of quality indices. Each observation which is normally a CCD image in FITS format has associated with it a header file 
which includes a complete set of data associated with the telescope system when the observation was made. It also includes an automatically generated quality index for the observations.

The quality index was originally designed for CCD photometry of stars. The programme searches for stars on the CCD image. It looks for objects which appear above the sky background and have a defined profile and roundness within certain limits. This works well with stars but most of the many tens of thousands of observing requests that the telescope has received are for planets, the moon, nebulae and galaxies. All of these receive a poor rating from the quality index even if they are excellent images.

The system has also demonstrated eavesdropping. This mode of operation allows the user to access the screens of the computers controlling the telescope. In this way the telescope control computers including Point and the image processing computer Image can be monitored over the Internet.

This mode of operation can be linked to detailed support pages on the net which can prepare students for the observations. A project on the velocity of light could provide details of early ideas and theories with a discussion of the size of the solar system to give an introduction to Roemer's method for measuring the velocity of light. The students could be referred to earlier observations of the occultations of lo which enable the period to be determined and predictions of the time of occultation. They could then eavesdrop on observations of Jupiter watching for Io to appear or disappear. Similar programmes could be used for variable stars of different types or for quasars.

Eavesdropping has a further application which is relevant to professional astronomers. Large telescopes could encourage astronomers who win observing time on the telescopes to provide home pages detailing their research programme and the implications of the results they are hoping to get. The Internet can then be used to introduce students to the research programmes and allow students to eavesdrop on the instrument screens as the data comes in. In this way students could be involved with the excitement of professional observational astronomy. Some programmes would not be suitable, but of the 20 large telescopes in the world it is likely that on any one night a few of these would be suitable for Internet eavesdroppers.

The telescope can also be controlled directly over the Internet. This is mainly used for engineering developments by the Bradford Group and for Television programmes which illustrate its use by students from anywhere on the planet. With the poor weather at the Haworth site, direct control or eavesdropping are not viable options

\section{The Use of the Telescope for Education}

The Nuffield Research Project contains six on-line projects in astronomy for schools and a host of support material for class-room activities. The projects are associated with the UK National Curriculum and link into the Bradford Robot Telescope. The projects were originally provided in paper and magnetic form and are now available on the Internet and consist of about 300 pages.

The projects for years 7 to 11 cover:

- An Introduction to Astronomy

- The Earth in Space

- The Planets

- The Solar System

- The Moon

- The Galaxy and the Universe.

Research projects for years 12 and 13: there is teacher support material which includes:-

- The project student learning objectives associated with the NC

- Teacher guides for the assessment of the projects linking them to NC attainment targets

- The objectives associated with the use of the Internet for teaching

- The objectives associated with using a robotic telescope for teaching 
- Technical details of the computer set-up and access to the Internet

- Guidelines for the teachers in the use of time.

The Six Projects cover the range of student age and ability consistent with the National Curriculum. The projects are in a 'least-work' for the teacher form and directly relate to the National Curriculum with notes showing how and where they fit in with the NC. They include a variety of support including suggested activities, worksheets and a level of differentiation.

Each project is totally self contained; where necessary it uses service observations by the Bradford Robot Telescope to demonstrate an aspect of the learning objectives of the project. The materials supporting the project are complete, illustrated, ready to use and copy free.

There were ten schools with IBM compatible PC computers which were given software, technical support and funding to link them to the Internet. About 150 schools were circulated with the material and provided with a degree of technical support, and thousands of schools from all over the world have come in to use the projects and the telescope over the Internet.

Our conclusion from running the project was that no schools had the necessary bandwidth to enable a whole class to use the Internet together and the most effective use of the resource was to download it from the net to a local server. There the projects could be printed out on paper for use by the class or if the school had a networked classroom of IBM compatible PC computers they could simulate access to the Internet using the resource on the server. Where the students could access the Internet in their own time then each could submit their own observing request; normally the requests were class requests.

In spite of the telescope being on a very poor site, which with the English weather was unable to give the turnaround necessary for class observations, many students had a very positive experience from the project and attended the final pupil researcher discussion meeting clearly excited with the science and technology they experienced with this project.

\section{The Future Plans}

The Bradford Robot Telescope is a fully functional system built to evaluate and develop the technology. It is now clear that this technology can provide access to observational astronomy for millions of students. The telescope operates in response to requests submitted over the Internet from anywhere on the planet. It can operate in real time responding to the requests of a single observer or it can respond to many observation requests scheduling them at the most appropriate time. In either case the Internet allows for eavesdropping on these observations with no limit on the numbers of eavesdroppers.

In order to expand this vision it would be necessary to install robot systems at good sites in the northern hemisphere and in the southern hemisphere. For Europe, optimum observatory sites would be in the USA (Arizona and Hawaii), Japan and Australia. For the world, sites in the northern and southern hemispheres which are distributed as evenly as possible around the planet would give access to the world's students at any time of the day or night. It is not just a facility for the developed nations of the world. Many of the sites should be in the developing nations and with true partnerships it would introduce them to the advanced technologies of the robot systems and communications and enable some of their students to take advantage of these systems.

The Bradford Robot Telescope development has illustrated that in a modern robot system $80 \%$ of the cost is in the software. The Bradford Group wish to build partnerships with observatories on good sites, with funders who wish to be associated with the projects, with colleges who wish to give their students access to classroom observational astronomy. The Bradford Group wish to share their expertise and software. They have the design for a 1.2 metre telescope that can operate as a robot. They have the software and systems designs to convert most computer controlled telescopes into robot operation. They are currently updating their system to run with a Meade LX200. They can provide a customised Internet interface for any partner and provide for them a daily schedule for their telescope and email to their users when the observations have been made. 
There are 25 million school students in Europe over the age of 11 who in the next few years will have access to the Internet. In the developed world there are about 70 million such students. A commercial partner will be associated by these students with an exciting programme linking them into a futuristic system to observe the Universe. It is well worth the few millions that it will cost to copy the Bradford systems and provide them for the school students of the world.

The Bradford Robotic Telescope demonstrates that practical astronomy is within the reach of students of all ages, directly from the classroom. No longer will it be necessary to wait for the dark and the weather. The link to the Intemet can transport the students to telescopes on good sites on the dark side of the earth and provide the impression of being in the control room 10 metres from the telescope. It is not the same as having your own telescope outside at night but for millions of young people it will inspire them with astronomy and the sciences, engineering and technology that support it.

\section{REFERENCES}

Mitchell, E.W.J., 1989, The Ground Based Plan - A Plan for Research in Astronomy and Planetary Science by Ground Based Techniques SERC Swindon ISBN 187066910 X.

Dunlap, J.C.\& Grabinger, R.S., (1996) Rich Environments for Active Learning FTP://ithaca.icbl.hw.ac.uk/pub/nato_asi/rsq1.rtf.gz

BoK, B.J. 1955 Size and Type of Telescope for a Photoelectric Observatory The Astronomical Journal (Astronomical Photoelectric Conference - Flagstaft Arizona 1953) Vol 60. pp31-32.

REDDISH, V.C., 1966 Sky and Telescope Vol 32. pp124.

Whitford, J. 1966 National Academy of Sciences - National Research Council Publication No 1234. Ground Based Astronomy-A Ten Year Programme.

BARUCH, J.E.F. 1992 Robots in Astronomy. Vistas in Astronomy Vol 35. pp399-438.

BARUCH, J.E.F. 1996 A Robot Telescope on the Internet, 1997 Year Book of Astronomy. Edited by Patrick Moore, Macmillan - London. 